\title{
Radial excitations of heavy-light mesons from QCD sum rules
}

\author{
P. Gelhausen, A. Khodjamiriana , A. A. Pivovarov, D. Rosenthal \\ Theoretische Physik 1, Naturwissenschaftlich-Technische Fakultät, Universität Siegen, 57068 Siegen, Germany
}

Received: 5 May 2014 / Accepted: 10 July 2014 / Published online: 13 August 2014

(C) The Author(s) 2014. This article is published with open access at Springerlink.com

\begin{abstract}
QCD sum rules are commonly used to predict the characteristics of ground-state hadrons. We demonstrate that two-point sum rules for the decay constants of charmed $\left(D^{(*)}, D_{s}^{(*)}\right)$ and bottom $\left(B^{(*)}, B_{s}^{(*)}\right)$ mesons can also be modified to estimate the decay constants of the first radial excitations, $D^{(*)^{\prime}}, D_{s}^{(*)^{\prime}}$ and $B^{(*)^{\prime}}, B_{s}^{(*)^{\prime}}$, respectively, provided the masses of these resonances are used as an input. For the radially excited charmed mesons we use available experimental data, whereas the masses of analogous bottom mesons are estimated from the heavy-quark limit. The decay constants predicted for the radial excitations of heavy-light pseudoscalar and vector mesons are systematically smaller than those of the ground states and we comment on the possible origin of this difference. Our results can be used in the sum rule calculations of heavy-to-light form factors and in the factorization approximations for nonleptonic $B$-meson decays where the decay constants of charmed mesons enter as input parameters.
\end{abstract}

\section{Introduction}

The spectrum of hadrons with a given spin-parity $\left(J^{P}\right)$ and flavor contains radial excitations, the sequential resonances heavier than the ground state. In the $N_{c} \rightarrow \infty$ limit of QCD a series of equidistant resonances is anticipated [1,2]. Models of equidistant states based on the resonance saturation of the two-point correlation functions are used to investigate quark-hadron duality and its violation [3-7]. Some recent applications to heavy-flavor decays can be found in $[8,9]$.

A clear identification of radial excitations on the background of hadronic continuum is a difficult experimental task. Usually these resonances are strongly coupled to the two- and three-hadron states from hadronic continuum, and these couplings generate large total widths. Note also that a strong mixing via intermediate continuum states can in

\footnotetext{
a e-mail: khodjamirian@tp1.physik.uni-siegen.de
}

principle significantly influence the pattern of the excited resonances, affecting their masses, widths and decay constants. It is therefore not surprising that radial excitations are well established [10] only for a few mesons. In this respect, the best studied are the neutral vector $\left(J^{P}=1^{-}\right)$mesons directly produced in $e^{+} e^{-}$annihilation, especially the heavy quarkonia. There are at least five (six) observed radial excitations $^{1}$ of the $J / \psi(\Upsilon(1 S))$ meson [10]. For the light-quark mesons some excited states are presented in [10], for example, $\rho(1450)$ and $\rho(1700)$ are identified as radial excitations of $\rho(770)$.

Relatively little is known about the radial excitations of heavy-light $D^{(*)}$ and $B^{(*)}$ mesons. On the experimental side, there are few observations of charmed resonances [10-13] whose quantum numbers and masses fit the expected properties of the radially excited states of $D, D^{*}$ and $D_{s}^{*}$, denoted here as $D^{\prime}, D^{*^{\prime}}$ and $D_{s}^{*^{\prime}}$, respectively. These resonances decay strongly to ground states and light mesons with a width in the ballpark of $100 \mathrm{MeV}$. A pseudoscalar charmedstrange meson $D_{s}^{\prime}$ was not yet established. Recent results of $\mathrm{LHCb}$ [13] and CDF [14] collaborations on the excited bottom mesons hint at $B^{\prime}$ - and $B^{*^{\prime}}$-states.

Recently the radial excitations of charmed mesons were discussed in connection with the semileptonic $B \rightarrow D^{\prime} \ell v_{\ell}$ decays [15-17], hence dynamical characteristics of these mesons are becoming a topical subject for heavy-flavor studies.

The properties of radially excited heavy-light resonances were predicted in various versions of the constituent quark model, starting from [18]; for a recent analysis, see e.g., [19].

\footnotetext{
1 The notion "radial excitations" stems from quantum mechanics, where it is used to denote the bound states obtained by solving the radial Schrödinger equation in a central potential. In the framework of the quarkonium potential model, the radially excited $2^{3} S_{1}$-state differs from the orbitally excited ${ }^{3} D_{1}$-state, both having the same $J^{P}=1^{-}$. In QCD, a hadron is a relativistic bound state with a certain valence quark content. Hence, here we count as radial excitations all resonances having the same flavor and $J^{P}$ as the ground state.
} 
Assuming the dominance of the valence quark-antiquark state and solving the bound-state problem for a certain quarkantiquark potential, one calculates the energy spectrum and the values of the wave functions at the origin, i.e., the masses and decay constants of radially excited mesons. However, the accuracy of the quark-model calculations is difficult to assess in QCD, since the relativistic quark-antiquark and gluon degrees of freedom beyond valence approximation are not explicitly included. Some other theoretical studies were presented in [20,21]. Quite recently, lattice QCD studies were performed to establish the properties of radially excited resonances. In particular, excited open-charmed mesons with $J^{P}=0^{-}, 1^{-}$have been studied in $[16,22,23]$.

In the QCD sum rule approach [24], a correlation function of two interpolating quark currents with a given flavor content and $J^{P}$ is calculated using the operator product expansion (OPE) in terms of QCD vacuum condensates. In the hadronic dispersion relation, obtained applying unitarity to this correlation function, all radially excited states, together with the continuum multi-hadron states with the quantum numbers of the interpolating currents are usually included into one hadronic spectral density whereas the ground-state contribution is isolated. The dispersion integral over the spectral density of excited and multi-hadron states is approximated applying the quark-hadron duality and introducing an effective threshold. After that the physical characteristics of the ground state are determined with a certain accuracy.

It is conceivable that a correlation function calculated in the spacelike region, via quark-hadron duality provides certain dynamical information not only on the ground state but on the whole spectrum of resonances. ${ }^{2}$ Indeed, as shown in [3-5], a correlation function, analytically continued to timelike momentum transfers, yields an infinite "comb" of equidistant poles. On the other hand, it is evident that the OPE with truncated power corrections can only provide a very limited information about hadronic states. In particular, the strong couplings of resonances to continuum states, their mixing and the resulting resonance widths are difficult to reproduce.

In this paper we consider as a study case the QCD sum rules for heavy-light currents, interpolating pseudoscalar and vector charmed and bottom mesons. The conjecture formulated above is addressed only to the first radial excitations of heavy-light mesons. We demonstrate that modifying QCD sum rules, it is possible to determine the decay constants of these states in addition to the ones of ground states. In what follows, we extensively use the results of our recent work [29] where the sum rules for pseudoscalar and vector heavy-light mesons were updated.

2 Attempts to describe the properties of excited states using finite energy sum rules, that is, attributing a finite duality interval to each sequential resonance, were made already quite some time ago; see [25-28].
The plan of the paper is as follows. In Sect. 2 we outline various procedures of constraining and/or estimating the decay constants of the first radially excited states using the sum rules. In Sect. 3 we present the numerical analysis. In Sect. 4 we summarize the results and discuss their possible applications.

\section{Including radial excitations in the sum rules}

In what follows, we consider QCD sum rules for the heavylight pseudoscalar $\left(J^{P}=0^{-}\right)$and vector $\left(J^{P}=1^{-}\right)$mesons, which are, respectively, obtained from the following correlation functions:

$\Pi_{5}(q)=i \int \mathrm{d}^{4} x e^{i q x}\left\langle 0\left|T\left\{j_{5}(x) j_{5}^{\dagger}(0)\right\}\right| 0\right\rangle$

and

$$
\begin{aligned}
\Pi_{\mu \nu}(q) & =i \int \mathrm{d}^{4} x e^{i q x}\left\langle 0\left|T\left\{j_{\mu}(x) j_{\nu}^{\dagger}(0)\right\}\right| 0\right\rangle \\
& =\left(-g_{\mu \nu} q^{2}+q_{\mu} q_{\nu}\right) \widetilde{\Pi}_{T}\left(q^{2}\right)+q_{\mu} q_{\nu} \Pi_{L}\left(q^{2}\right)
\end{aligned}
$$

where $j_{5}=\left(m_{Q}+m_{q}\right) \bar{q} i \gamma_{5} Q$ and $j_{\mu}=\bar{q} \gamma_{\mu} Q$ are the interpolating quark currents, $Q=c, b$ and $q=u, d, s$ are the quark fields with finite quark masses defined in $\overline{\mathrm{MS}}$-scheme. In (2), only $J^{P}=1^{-}$states contribute to the invariant amplitude multiplying the transverse kinematic structure, and we define $\Pi_{T}\left(q^{2}\right) \equiv q^{2} \widetilde{\Pi}_{T}\left(q^{2}\right)$. The decay constants of groundstate mesons $H=\{B, D\}$ and $H^{*}=\left\{B^{*}, D^{*}\right\}$ are defined in a standard way,

$$
\left\langle 0\left|j_{5}\right| H(q)\right\rangle=m_{H}^{2} f_{H}, \quad\left\langle 0\left|j_{\mu}\right| H^{*}(q)\right\rangle=m_{H^{*}} \epsilon_{\mu}^{\left(H^{*}\right)} f_{H^{*}},
$$

where $\epsilon_{\mu}^{\left(H^{*}\right)}$ is the polarization vector of $H^{*}$. The same definitions are used for the decay constants $f_{H^{\prime}}$ and $f_{H^{*}}$ of the radially excited states $H^{\prime}$ and $H^{*^{\prime}}$ with the masses $m_{H^{\prime}}$ and $m_{H^{*^{\prime}}}$, respectively.

The correlation functions are calculated at $q^{2} \ll m_{Q}^{2}$, using OPE which contains the perturbative part with $\mathcal{O}\left(\alpha_{s}^{2}\right)$ (NNLO) accuracy and the vacuum condensate contributions up to dimension $d=6$ :

$\Pi_{T(5)}^{\mathrm{OPE}}\left(q^{2}\right)=\Pi_{T(5)}^{(\mathrm{pert})}\left(q^{2}\right)+\Pi_{T(5)}^{\langle\bar{q} q\rangle}\left(q^{2}\right)+\Pi_{T(5)}^{\langle d 456\rangle}\left(q^{2}\right)$.

In the above, the quark condensate contribution (including the NLO terms calculated in [29]) and the sum of gluon, quark-gluon and four-quark condensate contributions with dimensions $d=4,5,6$ have, respectively, the indices $\langle\bar{q} q\rangle$ and $\langle d 456\rangle$. The expressions entering the OPE (4) can be found in [29] and we will not repeat them here for brevity. 
Turning to the hadronic representations of the correlation functions, we modify them, explicitly separating the first radial excitation from the spectrum of heavy-light states. The hadronic spectral densities of the correlation functions then get the following form:

$$
\begin{aligned}
\rho_{5}(s) \equiv & \frac{1}{\pi} \operatorname{Im} \Pi_{5}(s)=m_{H}^{4} f_{H}^{2} \delta\left(s-m_{H}^{2}\right) \\
& +m_{H^{\prime}}^{4} f_{H^{\prime}}^{2} \frac{\Gamma_{H^{\prime}} m_{H^{\prime}}}{\pi\left[\left(m_{H^{\prime}}^{2}-s\right)^{2}+\Gamma_{H^{\prime}}^{2} m_{H^{\prime}}^{2}\right]} \\
& +\tilde{\rho}_{5}^{h}(s) \theta\left(s-\left(m_{H^{*}}+m_{P}\right)^{2}\right)
\end{aligned}
$$

and

$$
\begin{aligned}
\rho_{T}(s) \equiv & \frac{1}{\pi} \operatorname{Im} \Pi_{T}(s)=m_{H^{*}}^{2} f_{H^{*}}^{2} \delta\left(s-m_{H^{*}}^{2}\right)+m_{H^{*^{\prime}}}^{2} f_{H^{*^{\prime}}}^{2} \\
& \times \frac{\Gamma_{H^{*^{\prime}}} m_{H^{*^{\prime}}}}{\pi\left[\left(m_{H^{*^{\prime}}}^{2}-s\right)^{2}+\Gamma_{H^{*^{\prime}}}^{2} m_{H^{*^{\prime}}}^{2}\right]} \\
& +\tilde{\rho}_{T}^{h}(s) \theta\left(s-\left(m_{H}+m_{P}\right)^{2}\right)
\end{aligned}
$$

where $P$ is the light pseudoscalar meson (pion or kaon). For the excited resonances we assume a Breit-Wigner (BW) form of the spectral density with a constant total width. The dependence on the width and the modification of the BW form with an energy-dependent width will also be investigated. The spectral densities $\tilde{\rho}_{5, T}^{h}(s)$ include the contributions of the excited states located above the first radial excitation and the continuum states. The latter start from the two-hadron thresholds $s=\left(m_{H^{*}}+m_{P}\right)^{2}\left(s=\left(m_{H}+m_{P}\right)^{2}\right)$ in the pseudoscalar (vector) channel. For pseudoscalar channels we take the decay $H \rightarrow H^{*} P$ as a physical process giving the threshold. For the vector channel we chose the process $H^{*} \rightarrow H P$. Note that the widths of the excited resonances $H^{(*)^{\prime}}$ are generated by their strong couplings to the continuum states, hence a part of the continuum contribution is effectively included in the radially excited resonance terms in the above spectral densities.

Our main assumption is that the semilocal quark-hadron duality approximation remains valid after isolating the excited state from the hadronic sum:

$$
\begin{aligned}
\tilde{\rho}_{5}^{h}(s) \theta\left(s-\left(m_{H^{*}}+m_{P}\right)^{2}\right) & =\rho_{5}^{\text {(pert) }}(s) \theta\left(s-\tilde{s}_{0}^{H}\right), \\
\tilde{\rho}_{T}^{h}(s) \theta\left(s-\left(m_{H}+m_{P}\right)^{2}\right) & =\rho_{T}^{\text {(pert) }}(s) \theta\left(s-\tilde{s}_{0}^{H^{*}}\right),
\end{aligned}
$$

where $\rho_{5, T}^{\text {(pert) }}(s)=(1 / \pi) \operatorname{Im} \Pi_{5, T}^{\text {(pert) }}(s)$ is the spectral density of the perturbative loop contributions to the OPE and $\widetilde{s}_{0}^{H^{(*)}}$ is the effective threshold. The latter parameter is expected to be larger than the one used in the conventional sum rules where only the ground state is separated from the hadronic sum. After applying the above duality ansatz and Borel transformation, the resulting sum rules are:

$$
\begin{aligned}
& f_{H}^{2} m_{H}^{4} e^{-\frac{m_{H}^{2}}{M^{2}}}+f_{H^{\prime}}^{2} m_{H^{\prime}}^{4} \int_{\left(m_{\left.H^{*}+m_{P}\right)^{2}}\right.}^{\infty} \mathrm{d} s e^{-\frac{s}{M^{2}}} \\
& \times \frac{m_{H^{\prime}} \Gamma_{H^{\prime}}}{\pi\left[\left(s-m_{H^{\prime}}^{2}\right)^{2}+m_{H^{\prime}}^{2} \Gamma_{H^{\prime}}^{2}\right]} \\
& =\widetilde{\Pi}_{5}^{(\text {pert })}\left(M^{2}, \widetilde{s}_{0}^{H}\right)+\widetilde{\Pi}_{5}^{\langle\bar{q} q\rangle}\left(M^{2}\right)+\widetilde{\Pi}_{5}^{\langle d 456\rangle}\left(M^{2}\right), \\
& f_{H^{*}}^{2} m_{H^{*}}^{2} e^{-\frac{m_{H^{*}}^{2}}{M^{2}}}+f_{H^{*^{\prime}}}^{2} m_{H^{*^{\prime}}}^{2} \int_{\left(m_{H}+m_{P}\right)^{2}}^{\infty} \mathrm{d} s e^{-\frac{s}{M^{2}}} \\
& \times \frac{m_{H^{*^{\prime}} \Gamma_{H^{*^{\prime}}}} \int^{\pi\left[\left(s-m_{H^{*^{\prime}}}^{2}\right)^{2}+m_{H^{*^{\prime}}}^{2} \Gamma_{H^{*^{\prime}}}^{2}\right]}}{=} \\
& \widetilde{\Pi}_{T}^{(p e r t)}\left(M^{2}, \widetilde{s}_{0}^{H^{*}}\right)+\widetilde{\Pi}_{T}^{\langle q q}\left(M^{2}\right)+\widetilde{\Pi}_{T}^{\langle d 456\rangle}\left(M^{2}\right),
\end{aligned}
$$

where the shorthand notation

$$
\widetilde{\Pi}_{T(5)}^{(\text {pert })}\left(M^{2}, s_{0}\right)=\int_{\left(m_{Q}+m_{q}\right)^{2}}^{s_{0}} \mathrm{~d} s e^{-s / M^{2}} \rho_{T(5)}^{\text {(pert) }}(s)
$$

is used and the Borel-transformed correlation function is denoted as $\widetilde{\Pi}_{T(5)}\left(M^{2}\right)$.

The masses and total widths of excited mesons $H^{\prime}$ and $H^{*^{\prime}}$ will be specified in the next section using experimental data on charmed states and heavy-quark symmetry relations. As usual in the sum rule analysis, one has to adopt an optimal interval of the Borel parameter values $\Delta M^{2} \equiv$ $\left\{M_{\min }^{2} \div M_{\max }^{2}\right\}$, where the lower (upper) boundary is chosen so that within this interval the OPE is reliable (the continuum contribution remains subleading). After that the above sum rules can be used to estimate the meson decay constants.

As a starting point we fix the decay constants of the ground states. We use the decay constants $f_{H}$ and $f_{H^{*}}$ obtained in [29] from conventional QCD sum rules (where the first excitation is included in the duality ansatz). Note that within uncertainties these values are in agreement with more accurate recent results from lattice QCD and also with the decay constants of charmed mesons extracted from experiment. Hence, lattice and/or experimental input values for the ground-state decay constants can equally well be used. After fixing the input values for the masses and ground-state residue, the two unknown parameters remain in each sum rule (9) or (10): the decay constant $f_{H^{(*)^{\prime}}}$ of the excited state we are interested in and the new effective threshold $\tilde{s}_{0}^{H^{(*)}}$. Putting the latter threshold to infinity, one immediately obtains the upper bound for $f_{H^{\prime}}$ or $f_{H^{*^{\prime}}}$. This bound is based on the positivity of all contributions to the hadronic spectral density and is independent of the duality approximation.

To employ the sum rules (9) and (10), while fixing the ground-state decay constants, one could try to follow the 
standard procedure (see e.g., [29]): adjusting the effective threshold to the mass of the excited state. The mass squared of the resonance can be calculated by dividing the sum rule differentiated over $\left(-1 / M^{2}\right)$ by the original sum rule. However, this procedure demands a very accurate knowledge of the excited meson mass, and practically only works in the zero width approximation when the spectral density of the excited state reduces to a delta function. Hence, in our numerical analysis presented below we use two different procedures:

(I) The decay constants $f_{H^{(*)}}$ and $f_{H^{(*)^{\prime}}}$ and the effective threshold $\tilde{s}_{0}^{H^{(*)}}$ are fitted simultaneously. To this end for each separate heavy-light channel, we minimize the squared difference between the 1.h.s. and r.h.s. of the sum rule summed over several points within $\Delta M^{2}$. E.g., for the pseudoscalar heavy-light meson channel we fit the values $f_{H}, f_{H^{\prime}}$ and $\widetilde{s}_{0}^{H}$ from

$$
\begin{aligned}
& \sum_{i} \mid f_{H}^{2} m_{H}^{4} e^{-\frac{m_{H}^{2}}{M_{i}^{2}}}+f_{H^{\prime}}^{2} m_{H^{\prime}}^{4} e^{-\frac{m_{H^{\prime}}^{2}}{M_{i}^{2}}} \\
& -\left.\left[\widetilde{\Pi}_{5}^{\text {(pert) }}\left(M_{i}^{2}, \tilde{s}_{0}^{H}\right)+\widetilde{\Pi}_{5}^{\langle\bar{q} q\rangle}\left(M_{i}^{2}\right)+\widetilde{\Pi}_{5}^{\langle d 456\rangle}\left(M_{i}^{2}\right)\right]\right|^{2}=\min ,
\end{aligned}
$$

where for brevity the width of the excited state is neglected being included in the numerical analysis. A similar minimization procedure is used for the vector meson channel.

(II) The ground-state contribution is eliminated from the sum rule. This is done, multiplying the correlation function by the factor $\left(m_{H^{(*)}}^{2}-q^{2}\right)$ before applying the Borel transformation. An equivalent procedure is to act with the differential operator $\left[\frac{\mathrm{d}}{\mathrm{d}\left(1 / M^{2}\right)}+m_{H^{(*)}}^{2}\right]$ on the sum rules (9) and (10). The correlation function is accordingly modified, so that the perturbative part (11) contains an additional factor $\left(m_{H^{(*)}}^{2}-s\right)$ under the integration. The resulting sum rule relations are then used to fit the decay constant of the excited state and the effective threshold with the minimization similar to (12) where now only the excited-state contribution is present.

We emphasize that the method used in this paper goes beyond the conventional sum rule technique. E.g., in the procedure (I) described above, the two decay constants and effective threshold are simultaneously fitted to the Boreltransformed correlation function. Hence, cautiously, one cannot exclude that a "systematic" uncertainty related to the quark-hadron duality is larger than in the usual sum rules. Still, due to the positivity of the hadronic spectral function, a cancelation between ground- and excited-state contributions in the sum rule cannot take place, hence a significantly biased estimate of decay constants is excluded. Furthermore, an important indication of the reliability is provided if both procedures (I) and (II) reproduce reasonably close values for the decay constant of a radially excited state.

\section{Numerical estimates}

The input parameters in the OPE on the r.h.s. of the sum rules (9) and (10) include the quark masses, strong coupling and condensate densities. We adopt the same values as in Table I of [29] where one can find the detailed discussion and relevant references. In particular, we use the $\overline{\mathrm{MS}}$ values of the quark masses: $\bar{m}_{b}\left(\bar{m}_{b}\right)=4.18 \pm 0.03 \mathrm{GeV}$, $\bar{m}_{c}\left(\bar{m}_{c}\right)=1.275 \pm 0.025 \mathrm{GeV}, \bar{m}_{s}(2 \mathrm{GeV})=95 \pm 10 \mathrm{MeV}$, the strong coupling $\alpha_{s}\left(M_{Z}\right)=0.1184 \pm 0.0007$, and the quark condensate density $\langle\bar{q} q\rangle(2 \mathrm{GeV})=-\left(277_{-10}^{+12} \mathrm{MeV}\right)^{3}$. We adopt the same default renormalization scale $\mu=1.5$ $\mathrm{GeV}(\mu=3 \mathrm{GeV})$ for the $c$-quark ( $b$-quark) correlation function as in [29] allowing further to vary it within the intervals $1.3 \mathrm{GeV} \div 3 \mathrm{GeV}(3 \mathrm{GeV} \div 5 \mathrm{GeV})$. The interval of the Borel parameter squared used in the sum rules for charmed mesons is $M^{2}=2.5 \div 3.5 \mathrm{GeV}^{2}$. We also vary it to $M^{2}=2.0 \div 3.0$ $\mathrm{GeV}^{2}$ and $M^{2}=3 \div 4 \mathrm{GeV}^{2}$ in order to estimate the related uncertainty of the results. For the bottom mesons we use $M^{2}=6.0 \div 8.0 \mathrm{GeV}^{2}$ as a default interval, shifting it to $M^{2}=5.5 \div 7.5 \mathrm{GeV}^{2}$ and $M^{2}=6.5 \div 8.5 \mathrm{GeV}^{2}$ for uncertainty estimates. The default Borel intervals still satisfy the criteria mentioned in the previous section although they are shifted to somewhat larger values with respect to the intervals used in [29]. This is done on purpose in order to enhance the contributions of the excited states.

In the hadronic part of the sum rule the masses and total widths of the three excited charm mesons are taken from experiment [10] and collected in Table 1. In particular, let us mention that the BaBar collaboration [11] observed two candidates for the radially excited charmed mesons: $D$ (2550) with $J^{P C}=0^{-}$and $D^{*}(2600)$ with $J^{P C}=1^{-}$. The charmed-strange radially excited state $D_{s}^{* \prime}(2700)$ with $J^{P}=1^{-}$was observed by several experiments $[10,12,13]$. To specify the mass of the remaining charmed-strange pseudoscalar meson we use an estimate

$m_{D_{s}^{\prime}}-m_{D_{s}} \approx m_{D^{\prime}}-m_{D}$,

relying on the $\mathrm{SU}(3)_{f l}$ symmetry for the excitation energy. For the radially excited $B$-mesons it is conceivable to apply simple relations valid in the heavy-quark limit:

$m_{B_{(s)}^{(*)^{\prime}}}-m_{B_{(s)}^{(*)}} \approx m_{D_{(s)}^{(*)^{\prime}}}-m_{D_{(s)}^{(*)}}$.

The estimated masses of excited hadrons are shown underlined in Table 1 and compared to the quark-model estimates from Ref. [19]. Note that quark-model predictions are for excited charmed mesons systematically larger than the available experimental values and for bottom mesons systematically smaller than the masses estimated from heavy-quark symmetry relations. The accuracy of symmetry relations for $B$-mesons is expected to be higher than for charmed mesons since the corrections are of $\mathcal{O}\left(1 / m_{Q}\right)$. The recently observed 
Table 1 Masses and total widths of the first radially excited heavy-light mesons and the mass shifts with respect to the ground-state mesons. The underlined masses and widths are our estimates for the yet unobserved resonances. The quark-model predictions [19] are shown in the last column

\begin{tabular}{|c|c|c|c|c|}
\hline$H^{(*)^{\prime}}$ & $m_{H^{\prime}}[\mathrm{MeV}]$ & $\Gamma_{H^{(*)^{\prime}}}[\mathrm{MeV}]$ & $m_{H^{(*)^{\prime}}}-m_{H}[\mathrm{MeV}]$ & $m_{H^{(*)^{\prime}}}^{Q M}[\mathrm{MeV}]$ \\
\hline$D^{\prime}$ & $2539 \pm 8$ & $130 \pm 18 \quad[10,11]$ & $669 \pm 8$ & 2581 \\
\hline$D^{*^{\prime}}$ & $2612 \pm 6$ & $93 \pm 14 \quad[10,11]$ & $601 \pm 6$ & 2632 \\
\hline$D_{s}^{\prime}$ & $\underline{2618 \pm 50}$ & $100 \pm 50$ & $\underline{650 \pm 50}$ & 2688 \\
\hline$D_{s}^{*^{\prime}}$ & $2709 \pm 4$ & $117 \pm 13$ & $597 \pm 4$ & 2731 \\
\hline$B^{\prime}$ & $5929 \pm 50$ & & & 5890 \\
\hline$B^{*^{\prime}}$ & $\underline{5975 \pm 50}$ & $100+50$ & $650+50$ & 5906 \\
\hline$B_{s}^{\prime}$ & $6017 \pm 50$ & $100 \pm 50$ & $050 \pm 00$ & 5976 \\
\hline$B_{s}^{*^{\prime}}$ & $6065 \pm 50$ & & & 5992 \\
\hline
\end{tabular}

state $B$ (5970) [14] interpreted as an excitation with $J^{P}=1^{-}$, nicely coincides with $B^{*^{\prime}}$-meson predicted from (14) with an estimated mass $m_{B^{* \prime}}=5,975 \mathrm{MeV}$. Hence, the relations based on heavy-quark symmetry seem to be reliable. We add $\pm 50 \mathrm{MeV}$ uncertainty to the central values of the estimates (14) to allow for a very conservative error. Furthermore, for the yet unknown total widths we assume a rather broad interval between 50 and $150 \mathrm{MeV}$ which is in the ballpark of measured total widths of charmed mesons. Note that an accurate prediction of the total widths of radially excited states is a difficult task because several channels of strong (flavorconserving) decays with the corresponding strong couplings contribute. In future, when the masses, branching fractions and total widths of all radially excited heavy-light mesons will be measured, one can substantially refine the above input.

The results for the decay constants for heavy-light mesons predicted from QCD sum rules (9) and (10) are collected in Table 2. In the first column we quote the decay constants of ground-state mesons obtained in [29]. We use these constants as inputs while obtaining the upper bounds for the decay constants of excited states which are independent of duality assumption. These bounds are calculated putting $\widetilde{s}_{0}^{H^{(*)}} \rightarrow \infty$ in the sum rules (9) and (10). For each bound the maximal value is determined within the optimal Borel interval. To this value we also add the uncertainty obtained after varying the parameters in the sum rules. The resulting bounds are presented in the last column of Table 2. We see that the bounds are somewhat restrictive for the charmed mesons, but not for bottom mesons.

Our main numerical results obtained from the fit procedures (I) and (II) described in the previous section are also shown in Table 2. Remarkably, these two quite different procedures predict consistent values of the decay constants of excited mesons. Most importantly, the ground- state decay constants obtained from the fit (I) are very close to the ones obtained in [29] from conventional sum rules, providing a cross-check of our calculation and ensuring the validity of quark-hadron duality beyond the ground state.

The uncertainties quoted in Table 2 originate from: (a) the variation of all input parameters in the OPE; (b) the shift of the $M^{2}$ intervals as explained above; (c) the mean squared fit error (reflecting the uncertainty induced by duality threshold); (d) the variation of the masses of excited states and widths within the intervals shown in Table 1 . Note that we prefer a rather conservative estimate and do not account for correlations between separate uncertainties adding them all in quadrature.

The estimated uncertainties for the ground-state decay constants obtained here and in [29] are in the same ballpark. For radially excited states the uncertainties of decay constants are intuitively expected to be larger than for the ground states. In fact, the contributions of excited resonances to the Borel-transformed correlation function have a smaller but comparable exponential weight with respect to the ground state, therefore the uncertainty returned by fit procedures (I) and (II) can also be in the same ballpark, as for example in the case of $B^{\prime}$ meson. The actual outcome of our numerical analysis becomes more transparent in terms of relative uncertainties, that is, if one divides the total variation quoted in Table 2 by the value obtained at the central input. These relative uncertainties vary from about $12 \%$ for the $B^{\prime}$-meson up to $40 \%$ for the $D_{s}^{*^{\prime}}$ and $B_{(s)}^{*^{\prime}}$ mesons. Meanwhile, the corresponding uncertainties for all heavy-light ground states do not exceed $15 \%$. Note that the "systematic" uncertainty caused by quark-hadron duality approximation, which is difficult to quantify on the basis of the input parameter variations, can also be somewhat larger for the decay constants of the excited states. 
Table 2 Decay constants of charmed and bottom mesons obtained from QCD sum rules and the corresponding effective thresholds

\begin{tabular}{|c|c|c|c|c|c|c|c|}
\hline \multirow[t]{2}{*}{ Meson } & \multirow{2}{*}{$\begin{array}{l}\text { Ref. [29] } \\
f_{H^{(*)}}(\mathrm{MeV})\end{array}$} & \multicolumn{3}{|l|}{ Procedure (I) } & \multicolumn{2}{|l|}{ Procedure (II) } & \multirow{2}{*}{$\begin{array}{l}\text { Upper bound } \\
f_{H^{(*)^{\prime}}}(\mathrm{MeV})\end{array}$} \\
\hline & & $f_{H^{(*)}}(\mathrm{MeV})$ & $f_{H^{(*)^{\prime}}}(\mathrm{MeV})$ & $\tilde{s}_{0}^{H^{(*)}}\left(\mathrm{GeV}^{2}\right)$ & $f_{H^{(*)^{\prime}}}(\mathrm{MeV})$ & $\tilde{s}_{0}^{H^{(*)}}\left(\mathrm{GeV}^{2}\right)$ & \\
\hline$D^{\left({ }^{\prime}\right)}$ & $201_{-13}^{+12}$ & $194_{-6}^{+6}$ & $137_{-23}^{+10}$ & 7.24 & $138_{-22}^{+10}$ & 7.24 & 189 \\
\hline$D_{s}^{\left({ }^{\prime}\right)}$ & $238_{-23}^{+13}$ & $230_{-9}^{+7}$ & $143_{-31}^{+19}$ & 7.48 & $146_{-36}^{+12}$ & 7.49 & 219 \\
\hline$D^{*\left({ }^{\prime}\right)}$ & $242_{-12}^{+20}$ & $235_{-12}^{+25}$ & $182_{-27}^{+12}$ & 7.43 & $183_{-24}^{+13}$ & 7.44 & 275 \\
\hline$D_{s}^{*\left({ }^{\prime}\right)}$ & $293_{-14}^{+19}$ & $279_{-12}^{+21}$ & $174_{-45}^{+22}$ & 7.87 & $178_{-39}^{+20}$ & 7.88 & 265 \\
\hline$B^{\left({ }^{\prime}\right)}$ & $207_{-9}^{+17}$ & $200_{-10}^{+18}$ & $163_{-11}^{+10}$ & 36.75 & $166_{-10}^{+9}$ & 36.78 & 279 \\
\hline$B_{s}^{(\prime)}$ & $242_{-12}^{+17}$ & $234_{-11}^{+15}$ & $174_{-19}^{+19}$ & 37.72 & $178_{-17}^{+19}$ & 37.75 & 320 \\
\hline$B^{*\left({ }^{\prime}\right)}$ & $210_{-12}^{+10}$ & $208_{-21}^{+12}$ & $163_{-13}^{+54}$ & 36.70 & $165_{-12}^{+46}$ & 36.71 & 314 \\
\hline$B_{s}^{*\left(\left(^{\prime}\right)\right.}$ & $251_{-16}^{+14}$ & $244_{-26}^{+13}$ & $190_{-20}^{+67}$ & 38.58 & $194_{-18}^{+57}$ & 38.61 & 325 \\
\hline
\end{tabular}

Table 3 Separate uncertainties for decay constants of heavy-light excited mesons from the sum rules applying the procedure (I) ((II)). All numbers are in $\mathrm{MeV}$

\begin{tabular}{|c|c|c|c|c|c|}
\hline Meson & $\Delta_{\text {param }}$ & $\Delta_{M^{2}}$ & $\Delta_{\text {fit }}$ & $\Delta_{\Gamma}$ & $\Delta_{m_{H^{\prime}}}$ \\
\hline$D^{\prime}$ & $\stackrel{+9}{+21}\left(\begin{array}{c}+9 \\
-20\end{array}\right)$ & $\begin{array}{l}+5 \\
-10\end{array}\left(\begin{array}{l}+5 \\
-8\end{array}\right)$ & ${ }_{-1}^{+1}\left(\begin{array}{c}+1 \\
-1\end{array}\right)$ & ${ }_{-1}^{+1}\left(\begin{array}{c}+1 \\
-1\end{array}\right)$ & $< \pm 1(< \pm 1)$ \\
\hline$D_{s}^{\prime}$ & ${ }_{-27}^{+12}\left(\begin{array}{c}+7 \\
-29\end{array}\right)$ & ${ }_{-14}^{+7}\left(\begin{array}{l}+6 \\
-16\end{array}\right)$ & ${ }_{-1}^{+1}\left(\begin{array}{c}+2 \\
-2\end{array}\right)$ & ${ }_{-8}^{+8}\left(\begin{array}{l}+4 \\
-14\end{array}\right)$ & $\begin{array}{l}+9 \\
-4\end{array}\left(\begin{array}{c}+7 \\
-4\end{array}\right)$ \\
\hline$D^{*^{\prime}}$ & ${ }_{-24}^{+11}\left(\begin{array}{c}+10 \\
-22\end{array}\right)$ & ${ }_{-12}^{+8}\left(\begin{array}{c}+7 \\
-10\end{array}\right)$ & ${ }_{-2}^{+2}\left(\begin{array}{c}+3 \\
-3\end{array}\right)$ & ${ }_{-1}^{+1}\left(\begin{array}{l}+1 \\
-1\end{array}\right)$ & $< \pm 1(< \pm 1)$ \\
\hline$D_{s}^{*^{\prime}}$ & ${ }_{-34}^{+14}\left(\begin{array}{c}+13 \\
-31\end{array}\right)$ & ${ }_{-29}^{+15}\left(\begin{array}{l}+13 \\
-23\end{array}\right)$ & ${ }_{-5}^{+5}\left(\begin{array}{l}+7 \\
-7\end{array}\right)$ & ${ }_{-2}^{+2}\left(\begin{array}{l}+2 \\
-2\end{array}\right)$ & $< \pm 1(< \pm 1)$ \\
\hline$B^{\prime}$ & ${ }_{-8}^{+7}\left(\begin{array}{l}+7 \\
-7\end{array}\right)$ & ${ }_{-7}^{+5}\left(\begin{array}{l}+5 \\
-6\end{array}\right)$ & ${ }_{-1}^{+1}\left(\begin{array}{l}+2 \\
-2\end{array}\right)$ & ${ }_{-4}^{+4}\left(\begin{array}{c}+4 \\
-4\end{array}\right)$ & ${ }_{-1}^{+2}\left(\begin{array}{l}+1 \\
-1\end{array}\right)$ \\
\hline$B_{s}^{\prime}$ & ${ }_{-11}^{+11}\left(\begin{array}{c}+9 \\
-10\end{array}\right)$ & ${ }_{-10}^{+8}\left(\begin{array}{c}+7 \\
-8\end{array}\right)$ & ${ }_{-2}^{+2}\left(\begin{array}{l}+3 \\
-3\end{array}\right)$ & ${ }_{-10}^{+11}\left(\begin{array}{c}+11 \\
-10\end{array}\right)$ & ${ }_{-1}^{+9}\left(\begin{array}{c}+10 \\
-1\end{array}\right)$ \\
\hline$B^{*^{\prime}}$ & $\begin{array}{l}+53 \\
-12\end{array}\left(\begin{array}{c}+46 \\
-10\end{array}\right)$ & ${ }_{-4}^{+4}\left(\begin{array}{l}+3 \\
-3\end{array}\right)$ & ${ }_{-1}^{+1}\left(\begin{array}{c}+2 \\
-2\end{array}\right)$ & ${ }_{-4}^{+4}\left(\begin{array}{c}+4 \\
-4\end{array}\right)$ & ${ }_{-3}^{+5}\left(\begin{array}{l}+4 \\
-3\end{array}\right)$ \\
\hline$B_{s}^{*^{\prime}}$ & ${ }_{-15}^{+66}\left(\begin{array}{c}+56 \\
-13\end{array}\right)$ & ${ }_{-11}^{+8}\left(\begin{array}{c}+7 \\
-9\end{array}\right)$ & ${ }_{-2}^{+2}\left(\begin{array}{c}+3 \\
-3\end{array}\right)$ & ${ }_{-7}^{+7}\left(\begin{array}{c}+7 \\
-7\end{array}\right)$ & ${ }_{-1}^{+4}\left(\begin{array}{c}+3 \\
-1\end{array}\right)$ \\
\hline
\end{tabular}

In Table 3 we present the error budget of our predictions in more detail. The largest uncertainties originate from the renormalization scale and quark mass variation, added in quadrature together with other input parameters in the OPE and denoted as $\Delta_{\text {param }}$ in Table 3. The variations of decay constants introduced by the choice of the Borel window $\left(\Delta_{M^{2}}\right)$, fit procedure $\left(\Delta_{\text {fit }}\right)$, uncertain masses $\left(\Delta_{m_{H^{\prime}}}\right)$ and widths $\left(\Delta_{\Gamma}\right)$ of the excited resonances are smaller.

As an alternative to a constant total width for excited heavy-light mesons, one can also use an energy-dependent width taking into account the hadronic continuum threshold. To investigate the influence of this effect we have inserted $\sqrt{s} \Gamma_{H^{(*)^{\prime}}}(s)$ instead of $m_{H^{(*)}} \Gamma_{H^{(*)^{\prime}}}$ in the BreitWigner ansatz for spectral densities (5) and (6) where for the $s$-dependent width the model [30] has been adopted (see, e.g., also [31]), e.g., for $H^{*^{\prime}}$ :

$\Gamma_{H^{*^{\prime}}}(s)=\Gamma_{H^{*^{\prime}}} \frac{m_{H^{*^{\prime}}}^{2}}{s}\left(\frac{\lambda\left(s, m_{H}^{2}, m_{P}^{2}\right)}{\lambda\left(m_{H^{*^{\prime}}}^{2}, m_{H}^{2}, m_{P}^{2}\right)}\right)^{\frac{3}{2}}$.

In the above, $\lambda(a, b, c)=a^{2}+b^{2}+c^{2}-2 a b-2 b c-2 a c$ and the kinematical factor originates from the $p$-wave phase space of the decay $H^{*^{\prime}} \rightarrow H P$. The analogous formula is valid for the excited pseudoscalar mesons where the width is dominated by the $H^{\prime} \rightarrow H^{*} P$ decay. Altogether, the influence of the total width on the decay constants of excited mesons obtained from the sum rules is small. To give an example, the $D_{s}^{\prime}$-meson decay constant obtained form the fit procedure (I) for the central input is $f_{D_{s}^{\prime}}=143 \pm 8 \mathrm{MeV}$, where here the uncertainty corresponds to varying only the total width within the interval $100 \pm 50 \mathrm{MeV}$. This value shifts to $f_{D_{s}^{\prime}}=148 \pm 16 \mathrm{MeV}$ if the energy-dependent width (15) is used in the fit. Note that neglecting the total width of $D_{s}^{\prime}$ altogether yields $f_{D_{s}^{\prime}}=128 \mathrm{MeV}$, a relatively small change.

Importantly, as seen from Table 2, the decay constant of a heavy-light excited meson is predicted systematically smaller than for its ground state. To investigate the origin of this difference we present in Table 4 the results of the numerical procedure (I) where separate contributions to OPE are switched off. We notice that when only the perturbative contributions are left and condensate contributions are neglected, the decay constants become numerically closer.

This brings us to the conclusion that the nonperturbative effects, most of all the leading contribution of the 
Table 4 Decay constants of the $D$ and $D^{\prime}$ mesons from the sum rules with different approximations for OPE (procedure (I), central/default input)

\begin{tabular}{lll}
\hline OPE approximation & $f_{D}(\mathrm{MeV})$ & $f_{D^{\prime}}(\mathrm{MeV})$ \\
\hline Perturbative LO & 120 & 123 \\
Perturbative LO+NLO+NNLO & 150 & 177 \\
Perturbative $+\langle\bar{q} q\rangle(\mathrm{LO}+\mathrm{NLO})$ & 190 & 142 \\
Perturbative $+\langle\bar{q} q\rangle(\mathrm{LO}+\mathrm{NLO})+\langle d 456\rangle$ & 194 & 137 \\
\hline
\end{tabular}

quark condensate effectively redistribute the spectral density of the correlation function so that the ground-state contribution gets enhanced and the first radial excitation suppressed.

\section{Conclusion}

In this paper we have attempted to determine the decay constants of first radially excited heavy-light mesons. To this end, modified QCD sum rules were obtained, in which, in addition to the ground-state meson contribution also the excited state is included being separated from the rest of the hadronic spectral density. We applied two different procedures, one of them independent of the ground-state contribution. The results of both procedures are consistent with each other and with the standard sum rule calculation [29] of the groundstate decay constants. This consistency justifies the validity of the quark-hadron duality approximation beyond the ground-state hadron in the correlation function of heavy-light currents. In future, the same technique can be used for other excited hadrons with various flavor and spin-parity quantum numbers. Also studies of non-diagonal two-point correlation functions can be useful, with two different currents having the same quantum numbers but different quark-gluon content. Here, one will employ the conjecture that excited hadronic states have a larger coupling to quark-antiquark-gluon currents.

Our results reveal a relative suppression of the decay constants of the radially excited states with respect to the ground state. This difference can be traced to nonperturbative effects. In future, more precise data on radially excited charmed and bottom mesons will allow us to improve the accuracy of our predictions.

The decay constant of the excited charmed meson $D^{\prime}$ predicted here agrees within uncertainties with the recent lattice QCD result $f_{D^{\prime}}=117 \pm 25 \mathrm{MeV}$ obtained in [16]. On the other hand, we cannot confirm the estimate $f_{D^{*^{\prime}}} \sim 300 \mathrm{MeV}$ obtained in [15].

The results obtained in this paper can be used in several ways. First, it is possible to extend hadronic representations in various light-cone sum rules (LCSR) in order to try alternative patterns of quark-hadron duality, beyond oneresonance approximation. E.g., in the LCSR's for $B \rightarrow \pi$ form factors, in the channel of the $B$-meson interpolating current, one can include in the hadronic spectral density the term involving the first radial excitation $B^{\prime}$ and the latter involves the decay constant $f_{B^{\prime}}$. Furthermore, as already mentioned, one needs accurate information on the excited charmed states including their decay constants for the studies of $B \rightarrow D^{\prime}$ form factors [15,16]. A promising source of information on charmed resonances are nonleptonic decays of $B$ mesons to open-charmed final states (see also [16]). For three-body and four-body $B$ decays accurate Dalitz-plot analyses are among the primary goals of current $B$-physics studies. These analyses demand reliable resonance-saturation models including the contributions of radially excited opencharmed states. For the amplitudes of these modes factorization estimates include the decay constants multiplied by heavy-to-light form factors. To bring just one example, the amplitude of $\bar{B}^{0} \rightarrow \bar{D}_{s}^{*^{\prime}-} \pi^{+}$decay contributes to the final state of three-body decay $\bar{B}^{0} \rightarrow \bar{D}^{0} K^{-} \pi^{+}$. The factorizable part of $\bar{B}^{0} \rightarrow \bar{D}_{s}^{*^{\prime}-} \pi^{+}$is color-enhanced, being proportional to the product of the $B \rightarrow \pi$ form factor at the momentum transfer $q^{2}=m_{D^{*^{\prime}}}^{2}$ and $f_{D^{*^{\prime}}}$. For the latter, the result of this study can immediately be used.

Acknowledgments We thank Thorsten Feldmann for useful comments and Danny van Dyk for a discussion of the statistical analysis. This work is supported by DFG Research Unit FOR 1873 "Quark Flavour Physics and Effective Theories”, Contract No. KH 205/2-1.

Open Access This article is distributed under the terms of the Creative Commons Attribution License which permits any use, distribution, and reproduction in any medium, provided the original author(s) and the source are credited.

Funded by $\mathrm{SCOAP}^{3}$ / License Version CC BY 4.0.

\section{References}

1. G. 't Hooft, Nucl. Phys. B 72, 461 (1974)

2. G. 't Hooft, Nucl. Phys. B 75, 461 (1974)

3. M.A. Shifman, in Particles, Strings and Cosmology, ed. by J. Bagger et al., (World Scientific, Singapore, 1996). hep-ph/9505289

4. B. Blok, M.A. Shifman, D.-X. Zhang, Phys. Rev. D 572691 (1998) [Erratum-ibid. D 59 (1999) 019901]

5. M.A. Shifman, in At the frontier of particle physics, vol. 3, ed. by M. Shifman, pp. 1447-1494. hep-ph/0009131

6. I.I.Y. Bigi, M.A. Shifman, N. Uraltsev, A.I. Vainshtein, Phys. Rev. D 59, 054011 (1999)

7. S. Groote, J.G. Körner, A.A. Pivovarov, Phys. Rev. D 65, 036001 (2002)

8. M. Beneke, G. Buchalla, M. Neubert, C.T. Sachrajda, Eur. Phys. J. C 61, 439 (2009)

9. M. Beylich, G. Buchalla, T. Feldmann, Eur. Phys. J. C 71, 1635 (2011)

10. J. Beringer et al. [Particle Data Group Collaboration], Phys. Rev. D 86 (2012) 010001 and 2013 partial update for the 2014 edition

11. P. del Amo Sanchez et al. [BaBar Collaboration], Phys. Rev. D 82, 111101 (2010) 
12. J. Brodzicka et al. [Belle Collaboration], Phys. Rev. Lett. 100, 092001 (2008)

13. A. Poluektov, PoS HQL 2012, 004 (2012)

14. T.A. Aaltonen et al. [CDF Collaboration]. arXiv:1309.5961 [hepex]

15. F.U. Bernlochner, Z. Ligeti, S. Turczyk, Phys. Rev. D 85, 094033 (2012)

16. D. Becirevic, B. Blossier, A. Gerardin, A. Le Yaouanc, F. Sanfilippo, Nucl. Phys. B 872, 313 (2013)

17. J. Segovia, E. Hernández, F. Fernández, D.R. Entem, Phys. Rev. D 87, 114009 (2013)

18. S. Godfrey, N. Isgur, Phys. Rev. D 32, 189 (1985)

19. D. Ebert, R.N. Faustov, V.O. Galkin, Eur. Phys. J. C 66, 197 (2010)

20. A.M. Badalian, B.L.G. Bakker, Phys. Rev. D 84, 034006 (2011)

21. Y. Sun, Q.-T. Song, D.-Y. Chen, X. Liu, S.-L. Zhu. arXiv:1401.1595 [hep-ph]
22. D. Mohler, S. Prelovsek, R.M. Woloshyn, Phys. Rev. D 87(3), 034501 (2013)

23. G. Moir, M. Peardon, S.M. Ryan, C.E. Thomas, L. Liu, JHEP 1305, $021(2013)$

24. M.A. Shifman, A.I. Vainshtein, V.I. Zakharov, Nucl. Phys. B 147, 385 (1979)

25. N.V. Krasnikov, A.A. Pivovarov, Phys. Lett. B 112, 397 (1982)

26. N.V. Krasnikov, A.A. Pivovarov, N.N. Tavkhelidze, Z. Phys. C 19, $301(1983)$

27. A.L. Kataev, Phys. Atom. Nucl. 68, 567 (2005)

28. S.S. Afonin, A.A. Andrianov, V.A. Andrianov, D. Espriu, JHEP 0404, 039 (2004)

29. P. Gelhausen, A. Khodjamirian, A.A. Pivovarov, D. Rosenthal, Phys. Rev. D 88, 014015 (2013)

30. J.H. Kuhn, A. Santamaria, Z. Phys. C 48, 445 (1990)

31. C. Bruch, A. Khodjamirian, J.H. Kuhn, Eur. Phys. J. C 39, 41 (2005) 